\title{
Anhelos humanos como propuestas investigativas
}

\section{Human Yearnings as Research Proposals}

Camila Gonzalez

\section{Resumen}

El ser humano destaca por su particular condición de entre los demás animales conocidos. Como criatura pensante es la única, conocida, capaz de pensarse a sí misma y a su entorno, y aunque esta información resulta obvia dado la época en la que nos encontramos, eso no hace menos interesantes aquellos artículos que han surgido de dicha capacidad reflexiva. Esta investigación aborda temas variados que van desde el origen mismo de la existencia hasta el fin de esta, y en este caso seis artículos para abordar, en cuyo trasfondo es posible evidenciar cómo los primitivos anhelos humanos se abren paso a través de diversos campos de estudio y reflexión.

Palabras clave: Pensamiento; Reflexión; Existencia.

\section{Abstract}

The human being stands out for its particular condition from among the other known animals. As a thinking creature, it is the only one, known, capable of thinking about itself and its environment, and although this information is obvious given the time in which we find ourselves, that does not make any less interesting those articles that have emerged from this reflective capacity. This research addresses various topics that go from the very origin of existence to the end of it, and in this case six articles to address, in the background of which it is possible to 
demonstrate how primitive human yearnings make their way through various fields of study and reflection.

Keywords: Thought; Reflection; Existence.

El ser humano destaca por su particular condición de entre los demás animales conocidos. Como criatura pensante es la única, conocida, capaz de pensarse a sí misma y a su entorno, y aunque esta información resulta obvia dado la época en la que nos encontramos, eso no hace menos interesantes aquellos artículos que han surgido de dicha capacidad reflexiva. Los temas son tan variados que van desde el origen mismo de la existencia hasta el fin de esta, y en este caso seis artículos para abordar, en cuyo trasfondo es posible evidenciar cómo los primitivos anhelos humanos se abren paso a través de diversos campos de estudio y reflexión.

Los primeros artículos de los que se hablará serán: "La ciudad: ¿hábitat o zoológico humano?” y “¿Qué más da? - La estética en Jean-Luc Marion”2 los cuales giran en torno a un anhelo espiritual, tocando temas como el individualismo. Y luego, el enfoque estará en: "Bioética y Tecnociencia"3,

\footnotetext{
1 Jesús David Girado Sierra, "La ciudad: ¿hábitat o zoológico humano?", Escritos 26, no. 57 (2018): 389-406.

http://dx.doi.org/10.18566/escr.v26n57.a07

2 Stéphane Vinolo, "¿Qué más da? - La Estética en Jean-Luc Marion", Escritos 25, no. 54 (2017): 197-220. http://dx.doi.org/10.18566/escr.v25n54.a09

${ }^{3}$ Victor B. Penchaszadeh, "Bioética y tecnociencia", Escritos 24, no. 53 (2016): 433-47. https://doi.org/10.18566/scr.v24n53.a09
} 
“Inteligencia Artificial ¿Reemplazando al Humano en la Psicoterapia?”4 y "Divulgación y Formación en Nanotecnología: Un Puente Hacia la Bioética"5, aquellos que evocan una reflexión sobre el lado más lógico del ser humano, un anhelo más material, centrándose en un ámbito más científico. También se tocarán “Francisco ¿el papa del fin de la era constantiniana?”6, "La impronta bíblica de laudato si'. Diálogo a dos voces entre la encíclica y los inicios del génesis"7, "La bibliometría y las publicaciones en teología"8 e "Inculturación de la teología como ejercicio hermenéutico de apropiación: hipótesis desde el de vita moysis de Gregorio de Nisa"9.

La fenomenología de la ciudad es el eje del artículo "La ciudad: ¿hábitat o zoológico humano?", Girado Sierra en este escrito explora tesis en las que se habla de un hábitat humano donde reinan la desatención cortes, el cooperativismo competitivo y las relaciones instrumentales, entendiendo la ciudad como un producto de la naturaleza humana, incluso la máxima expresión de lo humano en

\footnotetext{
4 Jairo Esteban Rivera Estrada y Diana Vanessa Sánchez Salazar, "Inteligencia artificial ¿reemplazando al humano en la psicoterapia?", Escritos 24, no. 53 (2016): 271-91. https://doi.org/10.18566/escr.v24n53.a02

5 Joaquín Darío Tutor Sánchez, "Divulgación y formación en nanotecnología: un puente hacia la bioética", Escritos 24, no. 53 (2016): 483-506.

https://doi.org/10.18566/escr.v24n53.a12

${ }^{6}$ Dumar Iván Espinosa Molina, "Francisco ¿el papa del fin de la era constantiniana?", Cuestiones Teológicas 44, no. 102 (2017): 347-372.

https://doi.org/10.18566/cueteo.v44n102.a06

${ }^{7}$ Hernán Cardona Ramírez y Memo Angel, "La impronta bíblica de laudato si'. Diálogo a dos voces entre la encíclica y los inicios del génesis", Cuestiones Teológicas vol. 44, no. 102 (2017): 301-346. https://doi.org/10.18566/cueteo.v44n102.a05

8 Iván Darío Toro Jaramillo, "La bibliometría y las publicaciones en teología". Cuestiones Teológicas vol. 44, no. 102 (2017): 229-239. https://doi.org/10.18566/cueteo.v44n102.a01 ${ }^{9}$ Orlando Solano Pinzón, "Inculturación de la teología como ejercicio hermenéutico de apropiación: hipótesis desde el de vita moysis de Gregorio de Nisa". Cuestiones Teológicas vol. 43, no. 100 (2016): 333-357. https://doi.org/10.18566/cueteo.v43n100.a06
} 
tanto ser natural-cultural. Sin embargo, también explora la comprensión de la ciudad como un gran zoológico humano, donde conviven el individualismo, la ansiedad, el aburrimiento, el estrés, la represión y la tensión, cuestiones por las cuales los urbanitas podrían hallarse, cada vez más, en constante peligro de enloquecer.

Este artículo busca el análisis de ambas perspectivas, resaltando los puntos más interesantes y las características más destacables de cada. Al hablar de la ciudad como hábitat reconoce que "la ecología humana ha de ser un método útil a la hora de comprender diversas problemáticas sociales y sus relaciones con el sentido de los espacios o las representaciones que se ponen en disputa en la simbiosis hombre-territorio"10. Y a su vez utiliza la idea del zoológico planteado que "la historia humana es entonces el relato de la manera en que los seres humanos han construido zoológicos humanos cada vez más grandes, más complejos"11.

Este proceso de análisis lleva al autor a reconocer ambas tesis como posturas que deben ser tomadas en su sentido metafórico. De esta manera es posible hallar su mayor aporte al ámbito teórico, puesto que esto permite distinguir y destacar las realidades sociales a las que estas apuntan: la lucha despiadada por la supervivencia, resignificada gracias al sistema económico contemporáneo; el dominio de ciertos territorios privilegiados por parte de individuos catalogados como aptos, provocando la segregación, marginación y explotación de terceros; el

${ }^{10}$ Girado Sierra, "La ciudad: ¿hábitat o zoológico humano?", 399.

11 Girado Sierra, "La ciudad: ¿hábitat o zoológico humano?", 401. 
agravamiento de la tensión, la ansiedad y el aburrimiento, como consecuencias lógicas del confinamiento que se experimenta cotidianamente en la ciudad; la naturalización de las relaciones humanas impersonales, instrumentales e inauténticas, donde no se esperan vínculos afectivos, sino un trato superficial basado en unos mínimos contractuales; y finalmente, la resignación ante el constante padecimiento de un sentimiento de soledad.

Estos factores han configurado una vida nerviosa, llena de ansiedad, insatisfacción, indiferencia y miedo hacia el otro, lo que puede llevar a una exploración que permita dar con nuevos horizontes espirituales que le den algún tipo de confort al ser humano.

Una situación que es de esperarse ante la vida ansiosa que es generada por la ciudad que mencionaba Girado Sierra. Tal tipo de situaciones generan un anhelo con una connotación más espiritual, provocando el fenómeno mencionado anteriormente, y también el planteado por Vinolo en “¿Qué más da? - La estética en Jean-Luc Marion".

Este otro artículo es bastante denso, ya que desea mostrar la distinción que Jean-Luc Marion realiza entre fenómenos de derecho común y fenómenos saturados, a su vez presentando la diferencia entre los objetos construidos o los fenómenos constituidos por un sujeto. A partir de esto se explora la pintura como una experiencia fenoménica donde la mirada del espectador se somete al fenómeno, planteando la posibilidad de recibir aquello que se quiere ver. Además, ello permite mostrar cómo desde el arte, el proceso de la visibilidad es presentado como construcción del fenómeno que opera desde lo invisible. Cuestiones 
filosóficas que en última instancia pretender evidenciar cómo la reflexión artística de Jean-Luc Marion permite entrar en el corazón de su obra, observando cómo se puede modificar los conceptos de donación y de sujeto.

Basta con decir que uno de los puntos fundamentales de la fenomenología de Marion es el pensar los fenómenos con el concepto de donación, haciendo del sujeto el donatario y no el donante de estos. En torno a esta concepción Vinolo reflexiona y plantea que "lo que nos dan a ver las obras de arte... presenta características fenomenológicas radicalmente diferentes de la de los objetos... lo que nos da a ver Los girasoles de Van Gogh es cualitativamente diferente de lo que recibimos como visibilidad cuando miramos girasoles en un jardín o en un campo"12.

Así que la conexión que se forma entre espectador y espectado es muy diferente cuando se observa una cosa y no una obra artística, pues "el objeto da a medida que nosotros le damos, es decir, el objeto da a nuestra medida; cuando la obra de arte siempre da mucho más allá de lo que podemos recibir, es porque ningún don nuestro primero le dio el patrón de lo que debe y de lo que puede devolver"13. Este tipo de apreciación resulta, cuanto menos, interesante, en especial cuando Vinolo menciona al amor, atribuyéndole esa característica que lo hace visible mucho más de quien lo recibe que de quien lo da, del donatario que del donante.

\footnotetext{
12 Vinolo, "¿QUÉ MÁS DA? - LA ESTÉTICA EN JEAN-LUC MARION", 200.
}

13 Vinolo, "¿QUÉ MÁS DA? - LA ESTÉTICA EN JEAN-LUC MARION", 217. 
Esta reflexión no solo habla del amor, este es solo un ejemplo que permite comprender de mejor manera la estética y los fenómenos saturados, ya que estos funcionan de una manera similar. En el texto se logra concluir que Marion le da su debida importancia al saber dar, una actitud muy valorizada en nuestra sociedad, ya que representa los valores de generosidad, de redistribución, de abnegación, etc. los cuales parecen desafiar al individualismo al que invita la ciudad. Sin embargo, Vinolo no se limita a tal perspectiva, también nos recuerda que, tal vez, por ese mismo individualismo, sea aún más importante y difícil saber recibir.

No obstante, el ser humano no se limita a los anhelos de tipo espiritual, no busca solo ser mejor en dicho ámbito, también busca avanzar, por ejemplo, en los ámbitos científicos y tecnológicos. La revolución tecnocientífica actual ha hecho que sea necesario reconfigurar el concepto de persona y de ser humano, ya que en esta década los avances tecnocientíficos ponen en cuestión nuestra propia humanidad y traen nuevamente a la mesa la idea de Superhombre planteada por Nietzsche.

La tecnología ha hecho que crezca el deseo de modificar nuestra condición humana buscando la perfección de las capacidades físicas, intelectuales y psicológicas. Las diferentes ciencias y áreas del conocimiento han debido adaptarse a estos cambios tecnocientíficos, incluso la psicología, la cual ha tenido que agregar a su terminología palabras como cyberterapia y terapia de realidad virtual. El texto "Inteligencia Artificial ¿Reemplazando al Humano en la Psicoterapia?", siguiendo tal hilo, busca establecer si la utilización de una inteligencia artificial en psicoterapia contribuye al mejoramiento del ser humano, 
permitiendo así un progreso en su salud mental, o si, por el contrario, su uso constituye un riesgo mayor para la humanidad, enfrentándola a la posibilidad del desplazamiento de los terapeutas humanos por máquinas inteligentes.

A lo largo del escrito, Rivera Estrada y Sánchez Salazar, exponen los precedentes de las inteligencias artificiales, los debates en torno a esas, y finalmente se centran del uso de estas en la psicoterapia. En este punto, es posible evidenciar que el anhelo de avanzar en el ámbito científico viene acompañado del temor de crear algo que sea superior y que llegue a reemplazar a los humanos. Los autores, conscientes de ello, llegan a concluir que la meta no debe ser la de reemplazar a los humanos, sino la de darles herramientas, por lo que "la superación o reemplazo de los seres humanos por las inteligencias artificiales, no se daría en tanto pudieran definitivamente realizar las mismas labores, inclusive mejor, sino en tanto pudieran incorporar la moral y la ética en sus decisiones al actuar"14. Una reflexión muy humana, ligada al ámbito de la ciencia y la tecnología, que pertenece a un debate que apenas empieza, y cuyo futuro está lleno de asombrosas y esperanzadoras incertidumbres.

Penchaszadeh sirve para hablar de la moral y ética necesarias para evitar un futuro desesperanzador para los humanos. En su texto, "Bioética y Tecnociencia" menciona una gran cantidad de dilemas bioéticos que han surgido en los últimos decenios debido al desarrollo de la tecnociencia. Plantea que "es preocupante que la formación de los profesionales de salud mayoritariamente

${ }^{14}$ Rivera Estrada y Sánchez Salazar, "Inteligencia artificial ¿reemplazando al humano en la psicoterapia?", 289. 
ignora la importancia de la bioética secular fundamentada en los derechos humanos, que debiera ser el eje de la capacitación para prestar atención de salud"15, y para exponer este punto se centra en la genómica. Un campo algo nuevo en el ámbito científico, pero que plantea una variedad de opciones al proponer el uso de las tecnologías para modificar el ADN, obteniendo ciertos beneficios, como la prevención de enfermedades, y así acercándose a ese humano perfecto.

Para permitir a sus lectores comprender mejor los dilemas bioéticos que surgen a raíz de este campo, Penchaszadeh aborda los objetivos y métodos de la genómica en salud, los métodos de las investigaciones genéticas y sus sesgos, la bioética y bases de datos genéticos, la bioética y manejo de muestras genéticas, entre otros. Y a través de estos subtítulos va configurando un pensamiento ético que permite una concepción bio-psico-social más equilibrada de la naturaleza humana, lo que permitiría aprovechar en forma ética el desarrollo de la ciencia genómica para el bienestar y la salud humana. Y de la misma manera esta ética puede beneficiar el área de la psicología, como buscaban Rivera Estrada y Sánchez Salazar, y también puede aportar en el ámbito de la nanotecnología, el punto de discusión del texto "Divulgación y Formación en Nanotecnología: Un Puente Hacia la Bioética".

Tutor Sánchez dedica su artículo, tal como se puede deducir por su título, al análisis del papel de la divulgación y la formación en nanotecnología como un puente entre las nanociencias y las nanotecnologías; sus aplicaciones en la

\footnotetext{
${ }^{15}$ Penchaszadeh, "BIOÉTICA Y TECNOCIENCIA", 447-448.
} 
biología, la biotecnología, el sector de la salud y el sistema médico farmacéutico, y el objeto de estudio de la bioética, principalmente la visión de bioética global. Al igual que Penchaszadeh, el autor lleva al lector por un recorrido que le permita adquirir el contexto sobre lo que está hablando, por lo que inicia con una breve introducción a los conceptos básicos de la bioética, el paradigma de la relación ciencia-tecnología-sociedad, y la aportación del conocimiento científico y tecnológico general a la bioética.

En este caso el escritor hace una presentación conceptual breve de la nanotecnología, sus beneficios y sus riesgos, siguiendo un hilo similar a los textos de inteligencia artificial y bioética y tecnociencia, ya que en ambos casos se vio necesario hablar de pros y contras. Esta cuestión denota que, pese a que cada uno tiene su enfoque, tal tipo de problemáticas presentan un proceso similar en el pensamiento, haciendo que cada uno se pregunte por las mismas cuestiones. Tutor Sánchez también toma conciencia de la manera en la que está avanzando la ciencia debido a los anhelos humanos, y el mismo expone su anhelo al hablar de la nanotecnología y decir que "debido al carácter emergente y convergente de las nanotecnologías, estas constituyen un eslabón muy importante en la cadena interactiva del conjunto de las ciencias y tecnologías que hacen sus aportaciones al objeto de estudio de la bioética"16.

La biotecnología y la medicina, incluyendo la psicología, dejada de lado en tantas ocasiones, forman parte de la cultura científica que debe interactuar con la

16 Tutor Sánchez, "Divulgación y formación en nanotecnología: un puente hacia la bioética", 504. 
cultura humanística para dar lugar a la bioética como ciencia, para así asegurar el futuro sostenible de nuestro mundo. El artículo finaliza argumentando porqué puede considerarse la divulgación y la formación en nanotecnología un puente hacia la bioética, concluyendo que la cultura social en nanotecnologías sin lugar a duda contribuirá a lograr un mundo mejor.

El ser humano destaca por su particular condición de entre los demás animales conocidos. Aunque obvio, no resulta menos fascinante el cómo del ejercicio reflexivo del humano de pensarse a sí mismo exponga sus anhelos a través de tanta diversidad. Marion, aquel del que hablaba Vinolo, Girado Sierra, Tutor Sánchez, Sarrazín Martínez, Penchaszadeh, Rivera Estrada y Sánchez Salazar, son personas que a través de sus textos denotan reflexiones muy humanas, el espíritu del anhelo humano de ser mejor en cualquiera de sus ámbitos. 


\section{Bibliografía}

Cardona Ramírez, Hernán y Memo Angel. "La impronta bíblica de laudato si'. Diálogo a dos voces entre la encíclica y los inicios del génesis”. Cuestiones Teológicas vol. 44, no. 102 (2017): 301-346. https://doi.org/10.18566/cueteo.v44n102.a05

Espinosa Molina, Dumar Iván. "Francisco ¿el papa del fin de la era constantiniana?". Cuestiones Teológicas 44, no. 102 (2017): 347-372. https://doi.org/10.18566/cueteo.v44n102.a06

Girado Sierra, Jesús David. "La ciudad: ¿hábitat o zoológico humano?" Escritos 26, no. 57 (2018): 389-406. http://dx.doi.org/10.18566/escr.v26n57.a07

Penchaszadeh, Victor B. "Bioética y tecnociencia". Escritos 24, no. 53 (2016): 433-47. https://doi.org/10.18566/scr.v24n53.a09

Rivera Estrada, Jairo Esteban y Diana Vanessa Sánchez Salazar. "Inteligencia artificial ¿reemplazando al humano en la psicoterapia?" Escritos 24, no. 53 (2016): 271-91. https://doi.org/10.18566/escr.v24n53.a02

Solano Pinzón, Orlando. "Inculturación de la teología como ejercicio hermenéutico de apropiación: hipótesis desde el de vita moysis de Gregorio de Nisa". Cuestiones Teológicas vol. 43, no. 100 (2016): 333-357. https://doi.org/10.18566/cueteo.v43n100.a06 
Toro-Jaramillo, Iván Darío. "La bibliometría y las publicaciones en teología". Cuestiones Teológicas 44, no. 102 (2017): 229-239. https://doi.org/10.18566/cueteo.v44n102.a01

Tutor Sánchez, Joaquín Darío. "Divulgación y formación en nanotecnología: un puente hacia la bioética". Escritos 24, no. 53 (2016): 483-506. https://doi.org/10.18566/escr.v24n53.a12

Vinolo, Stéphane. "¿Qué más da? - La Estética en Jean-Luc Marion". Escritos 25, no. 54 (2017): 197-220. http://dx.doi.org/10.18566/escr.v25n54.a09 